\title{
Selected aspects of proper integration between ERTMS/ETCS on-board and trackside devices.
}

\author{
Dominik Adamski , Andrzej Białon, and Zwadka Łukasz \\ Railway Research Institute, 04-275 Chłopickiego 50, Poland
}

\begin{abstract}
Achieving the interoperability of the European rail system requires many measures to unify technical solutions as well as regulations in each Member State. However, there is a possibility of some incompatibilities between individual subsystems despite generating them in accordance with unified applicable requirements. It is possible that the interoperable rolling stock will not be able to move freely over the interoperable railway line due to some incompatibilities and differences in the versions of the installed firmware in the ERTMS/ETCS system devices. In connection with the above the correct integration of rail vehicles with track-side equipment should be examined by means of tests under operating conditions. The article presents compliance tests of the correct integration of the on-board subsystem with the track-side subsystem which are carried out by the Railway Research Institute.
\end{abstract}

\section{Introduciton}

Over the years railway systems in particular Member States of the European Union have been developed independently using diversified technical solutions. The diversification of the technical rules, power supply, signaling, etc. has prevented the free movement of people and goods through the Member States. Therefore, it was decided to draw up the so-called White Paper 'Roadmap to a single European transport area towards a competitive and resource efficient transport system' where harmonization of technical requirements was considered to be the key issue in rail transport. The main goal was to lead to a situation in which trains will be able to cross the borders of European countries freely without stopping, on the contrary as it is today. In order to reach this goal it is necessary to achieve interoperability of the European rail system which will consequently lead to the creation of a single European railway area. This requires the elimination of a number of technical, legal and administrative obstacles. Thus, the railway system has been divided into structural subsystems [2]: infrastructure, energy, the controlcommand and signalling and rolling stock as well as functional subsystems: maintenance, operation and traffic management and telematics applications for passenger transport and freight transport. This division allowed drawing up specific Technical Specifications for Interoperability (TSIs) for particular subsystems, i.e. the requirements that must be met in order to achieve full technical harmonization of the railway. Dedicated to the relevant subsystems, the TSIs have the same structure, where the essential requirements, basic parameters, interfaces with other subsystems, the scope of necessary checks and inspections in order to obtain $\mathrm{EC}$ verification certificates, etc. are described.

The article focuses on issues related to the structural control-command and signalling subsystem implemented by the Commission Regulation (EU) No. 2016/919 of 27 May 2016 on the technical specification for interoperability within the control-command and signalling subsystems of the rail system in the European Union, hereinafter referred to as "TSI CCS "(from control-command and signalling) [1].

According to the provisions of Directive 2008/57/EC of June 17, 2008 command and signalling subsystem is described as: "All the equipment necessary to ensure safety and to command and control movements of trains authorised to travel on the network". Moreover [2] divides command and signalling subsystem into two separate subsystems i.e. " Control-Command and Signalling Onboard Subsystem " and " Control-Command and Signalling Track-side Subsystem". It is clearly visible that in spite of the common denominator "control-command and signalling ", both subsystems are independent assessment subjects for their EC verification by notified bodies [12]. It should also be remembered that apart from the above mentioned control-command and signalling layers of the subsystem so-called basic layer is also distinguished. It consists of track vacancy detection devices, turnouts, station, line equipment etc. The requirements for the basic layer are defined by national regulations and this is conditioned by the diversity of traffic regulations and the applied technical solutions in individual Member States like it is in Poland in [7]. The confirmation of meeting the TSI requirements by the subsystem is to receive the EC certificate of verification which, after carrying out the necessary checks and inspections is issued by the notified body $[9,12]$. 


\section{ERTMS System}

\subsection{Characteristics of the ERTMS system}

Regardless of the case under consideration, the European Rail Traffic Management System (ERTMS) is installed on interoperable railway vehicles and interoperable railway lines. ERTMS is defined to meet the TSI control-command and signalling requirements despite where it is implemented. The ERTMS system is classified as Class A and is divided into the European Train Control System (ETCS) and the Global System for Mobile Communications-Railways (GSM-R). The operation of the ETCS system is based on calculating and controlling the braking curves [4], [5]. The aforementioned curves depend on many factors relating both to the vehicle and to the track. When devising the system, it was assumed that these factors can be separated into track-dependent and vehicle-dependent. Thus, vehicle data includes information such as vehicle weight, maximum axle load, maximum speed, brake system parameters, etc.

Track data, as opposed to vehicle data (which is provided once before the train's departure), are received by the vehicle during the entire driving time. They change both in time (depending on the traffic situation) and in space (depending on the location of the vehicle). These data include, above all, an movement authority, which consists of the maximum distance that the vehicle can cover and the maximum speed as a function of the distance from the reference point. Together with the movement authority vehicle also receives another information which is determined by track-dependent factors affecting the braking curves counted by the system. Such information includes, for example, the track profile (up and down gradients), distances to neighboring balises and information about other track vehicle transmission channels.

ETCS systems in both on-board and track-side variants appear in three levels and depending on the requirements also several baselines. Baselines correspond to the version of the technical specifications designed and validated for particular project. TSI 2016/919 [1] indicates three sets of specifications that may be applied during EC verification. From 1 January 2019 the set of specifications only number 2 and 3 are valid for on-board subsystem. That means that since that date only vehicles equipped with baseline 3.4.0 and 3.6.0 are able to obtain EC certificate of verification excluding some specific cases with derogations. Differences between baselines consist in introducing additional functionalities clarifying the requirements as well as improving the stability of the system operation. Baseline 3.4.0 comparing to baseline $2.3 .0 \mathrm{~d}$ introduces new functions such as: Passive Shunting mode, Limited Supervision mode, level crossing not protected, new specification for the Non Leading mode, track conditions for the power supply, track conditions for sounding the horn, virtual balise cover etc. It is worth to mentioned that baselines backwards and forwards compatibility is defined in [6]. The rules for assigning the appropriate baseline for the GSM-R system are analogous to those applicable to ETCS and GSM-R system can be implemented in two versions: baseline 0 and baseline 1 . It is important to mention that project baseline is not related to the levels installed. The article focuses on onboard systems so detailed information regarding trackside subsystem baseline version management will be intentionally omitted.

ETCS is based on digital track-vehicle transmission. The transmission can be carried out by balises, short, medium or long loops, a digital radio channel or specialized transmission modules. Data describing the track and the vehicle are used to calculate static and dynamic speed profiles. The calculated profile is constantly compared to the current speed in the position function. The necessary location function is based on clearly distinguishable (through a unique number) and accurately located devices for point transmission (balises or loops). The control and supervision functions always operate according to the same rules regardless of the channel in which information from the track was received.

\subsection{System diversification}

Depending on the specific project, both on-board and track-side equipment are provided by different manufacturers. In addition, individual hardware configurations may differ in software versions, versions of interoperability constituents or components used because a certain manufacturer may for example allow to use three types of modems or four types of sensors for odometry devices with their generic product [10]. Moreover, rolling stock manufacturers in consultation with manufacturers of on-board devices must jointly develop a dedicated ETCS/ERTMS system configuration specific to a particular vehicle type and the ETCS/ERTMS system itself must be properly implemented in order correctly communicate and interacts with train systems. ERTMS/ETCS trackside implementation is also subject to similar conditions. Challenges arise relating to the connection between the Radio Control Center (RBC) with relay interlockings (basic layer) or with neighbouring $\mathrm{RBC}$, especially if it was built by another manufacturer.

Despite the introduction of standardization and unification of requirements relating to the controlcommand and signalling subsystem many issues still need to be clarified. Tests carried out in laboratories in simulated conditions as well as manufacturer's operational tests do not provide sufficient information on the correct integration of on-board equipment of the subsystem with track side equipment. This issue was already mentioned at the very introduction of [1] where it was stated: "Even a successful certification process cannot always exclude that, when an on-board CCS subsystem interacts with a trackside CCS subsystem, one of the subsystems repeatedly fails to function or perform as intended under certain conditions. This may be due to deficiencies in the specifications, different interpretations, design errors or equipment being 
installed incorrectly. A more coordinated way to perform compatibility tests should be introduced in order to help operators to take appropriate decisions."

Therefore table 6.2 of the [1] clearly states the checks than need to be carried out when verifying the onboard subsystem regardless of the chosen evaluation module. One of the aspects that has to be assess is "Integration with Control-Command and Signalling Trackside Subsystems and other subsystems: tests under operational conditions". These checks must be carried out on the line under as many different operational conditions as reasonably possible e.g. line gradient, train speed, traction power, weather conditions etc. The scope of these tests should not include tests already carried out at earlier stages for example tests carried out on the interoperability constituents or in the laboratory.

\section{Tests of a proper integration of subsystems}

Due to arisen situation the need to verify the correct integration of both subsystems in operational conditions was noticed and individual EU Member States were required by [1] to develop operational test scenarios. These tests are intended to check proper operation of the railway system in situations relevant to the ETCS and GSM-R and to demonstrate that the subsystems under examination are compatible with each other. Test scenarios created by each country's National Safety Authority (NSA) are to be sent to the European Railway Agency (ERA) in order to create a consolidated version of scenarios valid throughout the EU community in the future [11]. Currently scenarios elaborated by these entities are used in each Member State separately. In Poland the body that defined the test scenarios was the Office of Rail Transport (UTK). They were published in the form of tables describing individual test cases along with possible driver's actions, expected system reaction and finally the behavior of the tested vehicle.

Every vehicle subject to EC verification, irrespective of the chosen evaluation module based on [3], must pass the tests of correct integration with the track-side subsystem. Such tests must be carried out by an entity having appropriate competences and qualifications. Most often it is a Notified Body (NoBo) or the Designated Body (DeBo) cooperating with it. The Railway Research Institute conducts tests on the compliance of on-board subsystems with track-side as NoBo or as DeBo depending on the function performed in the given EC verification process. A similar situation takes place in the case of verification of track-side subsystems for which operational scenarios dedicated to specific implementations on specific lines are also provided by Infrastructure Managers. Issues related to the testing of trackside subsystems are not covered by scope of the article and therefore they will not be discussed.

\subsection{Test results}

The integration tests of a rolling stock with track-side infrastructure are carried out based on UTK operational scenarios and a set of additional checks developed by the Railway Research Institute. Train test runs are performed in fixed conditions in order to obtain a specific and expected results. Particular attention should be paid to the integration of the ERTMS/ETCS system with the Class B system, as it is the scope of a specific application for a given subsystem of a vehicle in a given country. During the tests, the correctness of the vehicle's transitions between levels and operating modes is checked, the response to changing the length of the Movement Authority (MA), speed control, reaction to the failure of individual components, etc. All indications on the Driver-Machine Interface (DMI) as well as the signals of semaphores are recorded using digital cameras. In addition, in the case of level 2 tests, it is coordinated from the level of RBC, where a representative of the Railway Research Institute is also present.

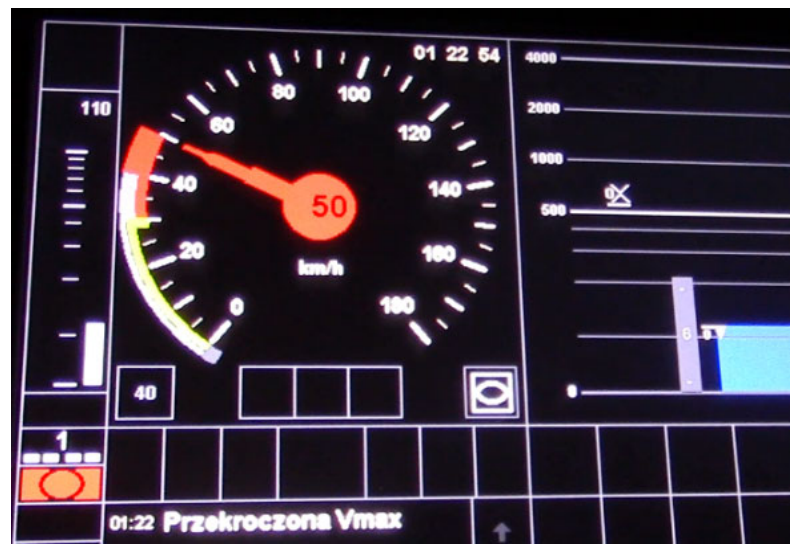

Fig. 1. DMI during test run [8].

The Juridical Recorder (JRU) is the source of all necessary data to evaluate the obtained results, in which all required train driving parameters are saved (see Fig.2). In addition, in order to verify the correctness of telegrams read from the balises and RBCs it is also possible to use the so-called Logs obtained from the European Vital Computer (EVC).

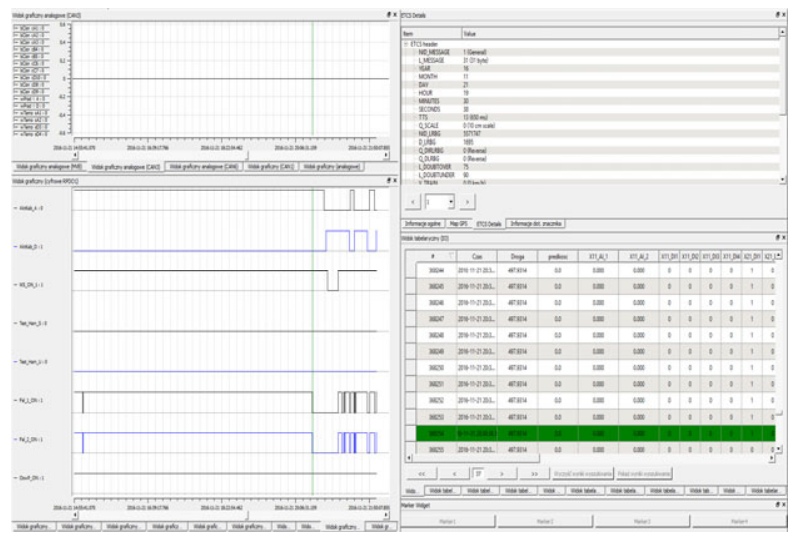

Fig. 2. Screen with raw data from JRU.

Data collected from the aforementioned sources, knowledge of the testing ground and visual observations made by qualified staff allow for an assessment of the correct integration of the on-board subsystem with track- 
side subsystem. In ambiguous cases, the assessment process is significantly extended, because it is necessary to analyze the error in detail in order to eliminate it. System errors that cause the TRIP - emergency vehicle stop , poor driving parameters (permitted speed reduced with no reason), problems with measuring the distance traveled, or measuring the distance from train antenna to the recently traveled balise are only a part of the difficulties encountered during discussed tests. In most cases, minor changes in the vehicle software are sufficient solution to the problems that arose but in extreme cases it is necessary to replace individual system components. Sometimes it is necessary to repeat whole test scope because necessary adjustments take time and solutions are not always obvious. For example, during the L2 tests, it turned out that the tested electric multiple traction unit that had two BTM components and EVC was incorrectly calculating distance between them. The result of this situation were problems with starting of a vehicle from known position after changing cab while $\mathrm{RBC}$ connection was still maintained. This issue was causing odometry errors and it was solved, after analyzing data from the vehicle and RBC telegrams, via software upgrade.

Observation of events and driving parameters in connection with the knowledge of individual subset requirements enables the analysis and subsequent assessment of the correct integration of on-board subsystem with trackside subsystem.

\section{Conclusion}

The issues of correct integration of the "Signalling Onboard Subsystem" with the "Signalling Trackside Subsystem" discussed in the article are an important element in the approval of the rolling stock. This is the final verification of the vehicle before obtaining approval for the putting into service. Detected errors and irregularities must be eliminated by introducing changes to the vehicle before issuing the $\mathrm{EC}$ verification certificate. Each ERTMS/ETCS on-board equipment manufacturer has an individual approach to the manner in which he implements the requirements of subsets and TSIs what in the final result may require the implementation of additional measures to ensure full compatibility of subsystems. Therefore because of variance in national signalling equipment (e.g. interlocking's), operational rules, different interpretations, possible errors in design etc. tests mentioned above shall be performed in order to demonstrate the technical compatibility of the considered subsystems.

The last but not least It should be remembered that laboratory and commissioning tests, due to their nature, do not allow to formulate the conclusion that the vehicle will properly cooperate with the infrastructure dedicated to it. Therefore commissioning checks should be extended by the aforementioned tests before placing rolling stock in the service. Probably in the future ERA will formulate a unified and consolidated test plan that will allow for unambiguous confirmation of compliance of the tested subsystems.

\section{References}

1. Commission Regulation (EU) 2016/919 of 27 May 2016 on the technical specification for interoperability relating to the 'control-command and signalling' subsystems of the rail system in the European Union

2. Commission Directive 2011/18/EU of 1 March 2011 amending Annexes II, V and VI to Directive 2008/57/EC of the European Parliament and of the Council on the interoperability of the rail system within the Community

3. Commission Decision (EU) 2010/713 of 9 November 2010 on modules for the procedures for assessment of conformity, suitability for use and EC verification to be used in the technical specifications for interoperability adopted under Directive 2008/57/EC of the European Parliament and of the Council

4. System Requirements Specification UNISIG SUBSET-026 version 2.3.0d

5. System Requirements Specification UNISIG SUBSET-026 version 3.4.0

6. System Requirements Specification UNISIG SUBSET-104 version 3.3.0

7. The list of the President of the Office of Rail Transport on the relevant national technical specifications and standardization documents, the use of which allows meeting the essential requirements relating to the interoperability of the rail system of 19 January 2017.

8. Adamski D., Ortel K. Problematyka wspótpracy poktadowego systemu ERTMS/ETCS z polska infrastruktura, Autobusy (6/2018)

9. Kycko M., Metodyka certyfikacji podsystemu sterowanie, Prace Naukowe Politechniki Warszawskiej, Warsaw (2016)

10. Pawlik M., Europejski System Zarządzania Ruchem Kolejowym, przegląd funkcji $i$ rozwiazań technicznych - od idei do wdrożeń $i$ eksploatacji, KOW, Warsaw (2015)

11. https://www.era.europa.eu/activities/european-railtraffic-management-system-ertms_en.

12. Gradowski P.: Upgrading the railway infrastructure technical parameters using the example of the Control-Command and Signalling subsystem with the EC verification certificate, Railway Reports, issue 182 (March 2019), ISSN 0552-2145, Railway Research Institute, Warsaw 2019, pp. 131-146 